\title{
Symmetry, Stereotypy, and Topography of Odorant Representations in Mouse Olfactory Bulbs
}

\author{
Leonardo Belluscio and Lawrence C. Katz \\ Howard Hughes Medical Institute and Department of Neurobiology, Duke University Medical Center, Durham, \\ North Carolina 27710
}

The molecular basis of vertebrate odorant representations has been derived extensively from mice. The functional correlates of these molecular features were visualized using optical imaging of intrinsic signals in mouse olfactory bulbs. Single odorants activated clusters of glomeruli in consistent, restricted portions of the bulb. Patterns of activated glomeruli were clearly bilaterally symmetric and consistent in different individual mice, but the precise number, position, and intensity of activated glomeruli in the two bulbs of the same individual and between individuals varied considerably. Representations of aliphatic aldehydes of different carbon chain length shifted systematically

The transform between molecular maps of odorant receptors and the physiological response to odors in the mammalian olfactory bulb has been deciphered with several approaches, including 2-deoxyglucose (2-DG) autoradiography (Sharp et al., 1975, 1977; Skeen, 1977; Stewart et al., 1979; Jourdan et al., 1980; Lancet et al., 1982; Leon et al., 1984; Johnson and Leon, 2000a,b) c-fos expression (Onoda, 1992; Guthrie et al., 1993; Sallaz and Jourdan, 1993), and functional magnetic resonance imaging (Yang et al., 1998; Xu et al., 2000). Optical imaging techniques, which have higher spatial and temporal resolution and can be used in living animals, have enabled visualization of odorant-evoked glomerular activation patterns in invertebrates and nonmammalian vertebrates (Cinelli et al., 1995; Friedrich and Korsching, 1997; Joerges et al., 1997; Faber et al., 1999). In mammals, optical imaging of intrinsic signals in the rat olfactory bulb has revealed detailed spatial patterns of glomerular activation representing different odorants, the concentration dependence of glomerular activation, and the molecular receptive ranges of specific glomeruli (Rubin and Katz, 1999; Uchida et al., 2000).

Although all species imaged to date provide valuable information on the general principles of organization of the initial stages of olfactory processing, the detailed molecular mapping of odorant receptors, their epithelial distribution, and their projections to the olfactory bulb have been accomplished primarily in mice (Ressler et al., 1993, 1994; Mombaerts et al., 1996; Wang et al., 1998). Several molecular features of the murine olfactory system make specific functional predictions. Axonal projections from all epithelial neurons expressing the same odorant receptor converge

Received Nov. 17, 2000; revised Jan. 4, 2001; accepted Jan. 4, 2001.

L.C.K is an Investigator in the Howard Hughes Medical Institute. L.B. is a Burroughs Wellcome Fellow in Neuroscience. We thank B. Rubin for his invaluable assistance with experiments and his comments on this manuscript.

Correspondence should be addressed to Leonardo Belluscio, Department of Neurobiology, Box 3209, Duke University Medical Center, Durham NC 27710. E-mail: belluscio@neuro.duke.edu.

Copyright (C) 2001 Society for Neuroscience 0270-6474/01/212113-10\$15.00/0 along a rostral-caudal strip of the dorsal bulb, indicating a functional topography of odorant representations. Binary mixtures of individual aldehydes elicited patterns of glomerular activation that were topographic combinations of the maps for each individual odor. Thus the principles derived from the molecular organization of a small subset of murine olfactory receptor neuron projection patterns-bilateral symmetry, local clustering, and local variability-are reliable guides to the initial functional representation of odorant molecules.

Key words: optical imaging; functional maps; glomeruli; odorant mixtures; aldehydes; olfactory

onto one or a few bilaterally symmetric glomeruli within the main olfactory bulb with limited variability between animals (Ressler et al., 1994; Vassar et al., 1994; Mombaerts et al., 1996; Strotmann et al., 2000). Olfactory receptors sharing the highest coding sequence identity bind structurally similar odorants (Malnic et al., 1999), and the neurons expressing these receptors project to neighboring glomeruli (Wang et al., 1998; Tsuboi et al., 1999; Johnson and Leon, 2000a,b; Uchida et al., 2000). Functionally these features predict that (1) odorants should activate bilaterally symmetric patterns of glomeruli in the two olfactory bulbs, (2) patterns elicited by a given odorant should be consistent between individuals, and (3) structurally related odorants should activate neighboring regions, forming identifiable maps.

To test these predictions, we used intrinsic signal imaging to define the functional representation of individual odorants. Furthermore, because most stimuli in the natural world occur as mixtures and are experienced in the presence of other odorants, we also began characterizing how odorant mixtures are represented at this early stage of processing. Consistent with the organization predicted from molecular mapping studies, we find that the activity maps in response to single odorants are bilaterally symmetric within an individual and remarkably similar between individuals. Maps of structurally related odorants occupy adjacent regions, suggesting a functional topography within the bulb. Moreover, mixtures evoke patterns of activity with symmetry and position that are predicted by the activity maps of the individual components.

\section{MATERIALS AND METHODS}

Animal preparation

Experiments were performed on 66 female C57BL/6 mice (12-20 weeks old). Mice were initially anesthetized with a mixture of ketamine (200 $\mathrm{mg} / \mathrm{kg}$, i.p.) and xylazine $(10 \mathrm{mg} / \mathrm{kg}$, i.p.). Mice were free breathing, and their anesthetic state was maintained with either isofluorane (1-3\%) or sevofluorane $(2-4 \%)$. Initial experiments were performed to ensure that the anesthesia gases alone did not evoke activity within the dorsal 
Figure 1. Discrete activated regions visualized with intrinsic signal imaging correspond to anatomical glomeruli. $A$, Blood vessel pattern overlying the imaged region visualized through thinned bone. Green asterisks indicate vessel landmarks. $B$, Olfactory map evoked by $1 \%$ propanal produced several active regions. Superimposed on the optical image are outlines of the glomerular pattern derived from $F$. The strongly activated regions are almost entirely confined to the anatomical borders of the glomeruli. $C$, Activity map from $B$ thresholded at 2 SDs, pseudocolored, and superimposed on the blood vessel pattern from $A$. Three distinct active regions the size and shape of anatomical glomeruli are strongly activated. $D$, Blood vessel pattern of imaged area used for subsequent alignment after removal of bone and dura; many of the same blood vessels are readily visible. $E$, Glomeruli revealed by vital dye staining with the voltage-sensitive dye RH414 and observed with epifluorescence microscopy. F, Outlines of individual glomeruli from $E$; the three activated glomeruli seen in $B$ and $C$ are highlighted. Scale bar, $200 \mu \mathrm{m}$.
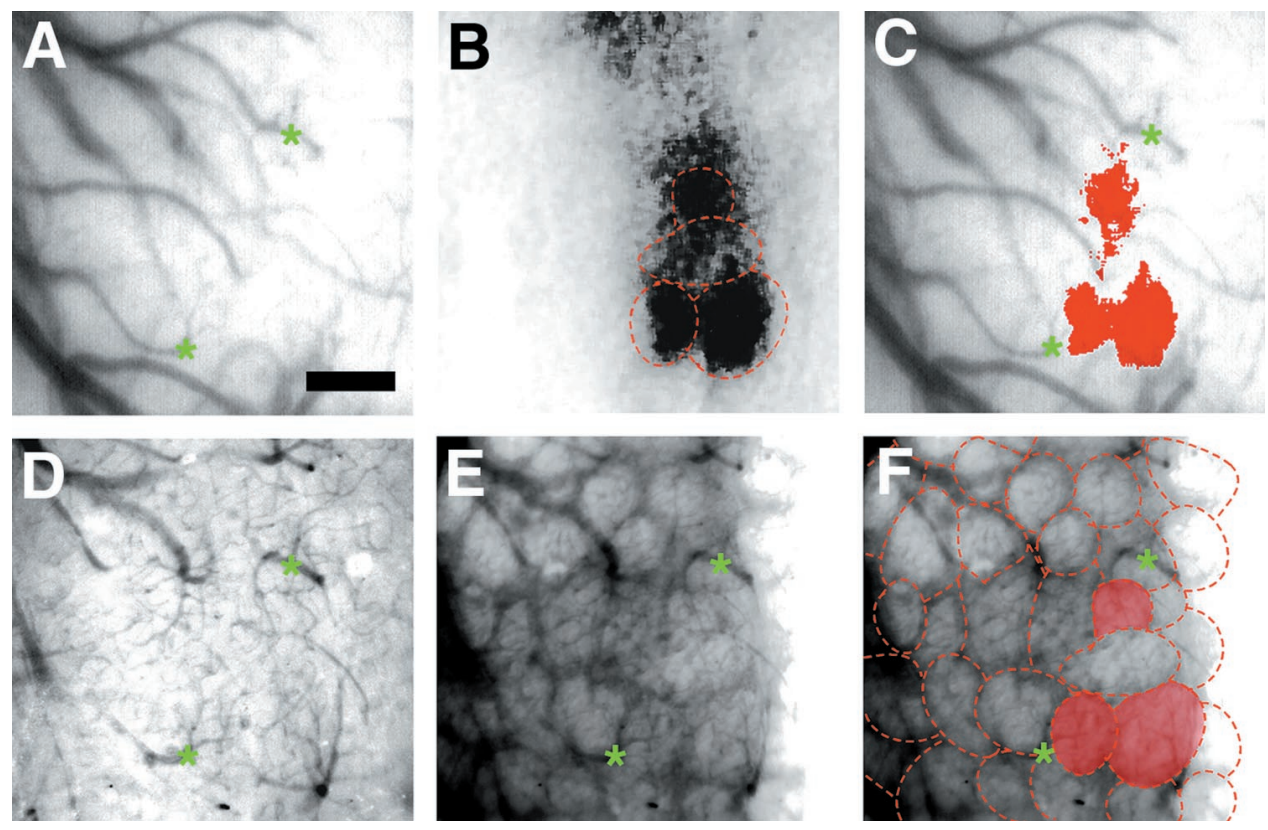

olfactory bulb. Similar results were obtained with both anesthetics. Once anesthetized, mice were placed in a stereotaxic apparatus, and the bone overlying the dorsal surface of both olfactory bulbs was thinned for imaging as described previously (Rubin and Katz, 1999). All mice used in this study were maintained in strict accordance with National Institutes of Health and institutional animal care guidelines.

\section{Intrinsic signal imaging}

Intrinsic signals were recorded using a commercially available imaging system (Imager 2001, Optical Imaging Inc.). Before each experiment, the surface blood vessel pattern was acquired under green light illumination $(546 \mathrm{~nm})$. Stimuli were delivered by placing a test tube filled with $1 \mathrm{ml}$ of an odorant (Sigma, St. Louis, MO, and Fluka, Buchs, Switzerland; at highest purity available) within $1 \mathrm{~cm}$ of the mouse's nose for $10 \mathrm{sec}$. Odorants were presented diluted in mineral oil (freshly prepared from stocks maintained under argon). Trials containing a single odorant stimulus and a mineral oil control were randomly interleaved and repeated 10 times with a $60 \mathrm{sec}$ interstimulus interval to minimize adaptation and habituation. Binary mixture experiments used three odorants (two individual odorants and a mixture) and a mineral oil control, and all stimuli were presented 20 times to increase signal-to-noise ratios. Video frames acquired under red $(630 \mathrm{~nm})$ illumination were summed, and differential odorant maps were constructed as described previously $(\mathrm{Ru}-$ bin and Katz, 1999). Images of odorant responses were initially visualized using IPLab (Scanalytics) and processed using a $5 \times 5$ pixel median filter to reduce high-frequency noise while maintaining spatial information. All final images were imported into Adobe Photoshop 5.5 for cropping and display.

\section{Vital dye staining}

After intrinsic imaging the thinned bone overlying the dorsal surface of the bulbs was removed, the dura was peeled away, and the surface of the bulbs was flushed with sterile oxygenated Ringer's solution. A small drop of $1 \mathrm{~mm}$ RH414 dye solution (Molecular Probes, Eugene, OR) was placed over the exposed bulbs and left in place for $5 \mathrm{~min}$. The dye solution was then removed, and the region was flushed continuously for $5 \mathrm{~min}$ with fresh Ringer's solution. The imaging chamber was replaced, and glomerular images were acquired as described (LaMantia and Purves, 1992; Bozza and Kauer, 1998).

\section{Image analysis}

Symmetry. For overlap symmetry analysis, images were imported into Metamorph (Universal Imaging, West Chester, PA), and the threshold was set at 2 SDs above the mean pixel value. To reduce noise, a symmetrical region of interest (ROI) was drawn around the perimeter of each bulb encompassing the entire exposed region of bulb but excluding the peripheral bone and midline vessels. This ROI was then used as a standardized mask and applied to each image identically. The image within the ROI was then duplicated, flipped horizontally, and superimposed on the original. To further improve accuracy, each superimposed maps was rotated $\pm 5^{\circ}$ to account for slight discrepancies in alignment while maximizing the pixel overlap. The number of active overlapping pixels was quantified and expressed as a percentage of the total number of active pixels within each map. Similar analyses were performed to compare image maps of two different odorants.

Centroid analysis. All centroid analysis was accomplished using IPLab (Scanalytics). Image maps were first thresholded at $>2$ SD above background, and the coordinate centers of each active peak were marked. A minimal straight line perimeter was drawn around all marked peaks, and the centroid of the region was determined. The degree of symmetry was then determined by calculating the ratios of the $x$ and $y$ coordinates from the left and right centroids. This was done for each map for all odors. The ratios were then averaged for each odor and compared. Data were processed and graphed using Microsoft Excel and imported into Adobe Photoshop for display.

Cluster analysis. To determine the consensus regions activated by different odorants, we collapsed the active centers for each odorant of 43 different maps into one composite image. A minimal perimeter was drawn around each group of odorant peaks to define the consensus region activated by an odorant. The area and centroid for each consensus region were then calculated and compared with the total area of bulb imaged and with the centroid coordinates of the opposite bulb.

\section{Mixtures}

All odorants were diluted to $1 \mathrm{ml}$ in mineral oil to a final concentration of either 1 or $2 \%$. Mice were then presented with each odorant randomly interleaved within the same experiment as described above. Images were collected, and values were stored as floating point values. Sets of images, each consisting of two single odor maps and a mixture map, were then analyzed for changes in map topography while controlling for intensity differences. Using MatLab, each image was read into a two-dimensional matrix containing the position and intensity value of each pixel. From the individual odor maps a predicted mathematical mixture image was then calculated as follows: each pixel from a single odor map was compared with the corresponding pixel in the other single odor map for intensity value. The two values were also compared with the value of the sum of the two pixels. The value of highest intensity (activity) was assigned to the mathematical mixture map. Using this method we could account for false signal cancellation. For example, if one map had higher signal in one region than a second map, the calculated sum (mixture) map would have at least the predicted value of the individual map, rather than the two signals canceling each other as would happen if the two images were simply summed. The mathematical image was then subtracted from the experimentally collected mixture image, producing a difference image. The difference image was 

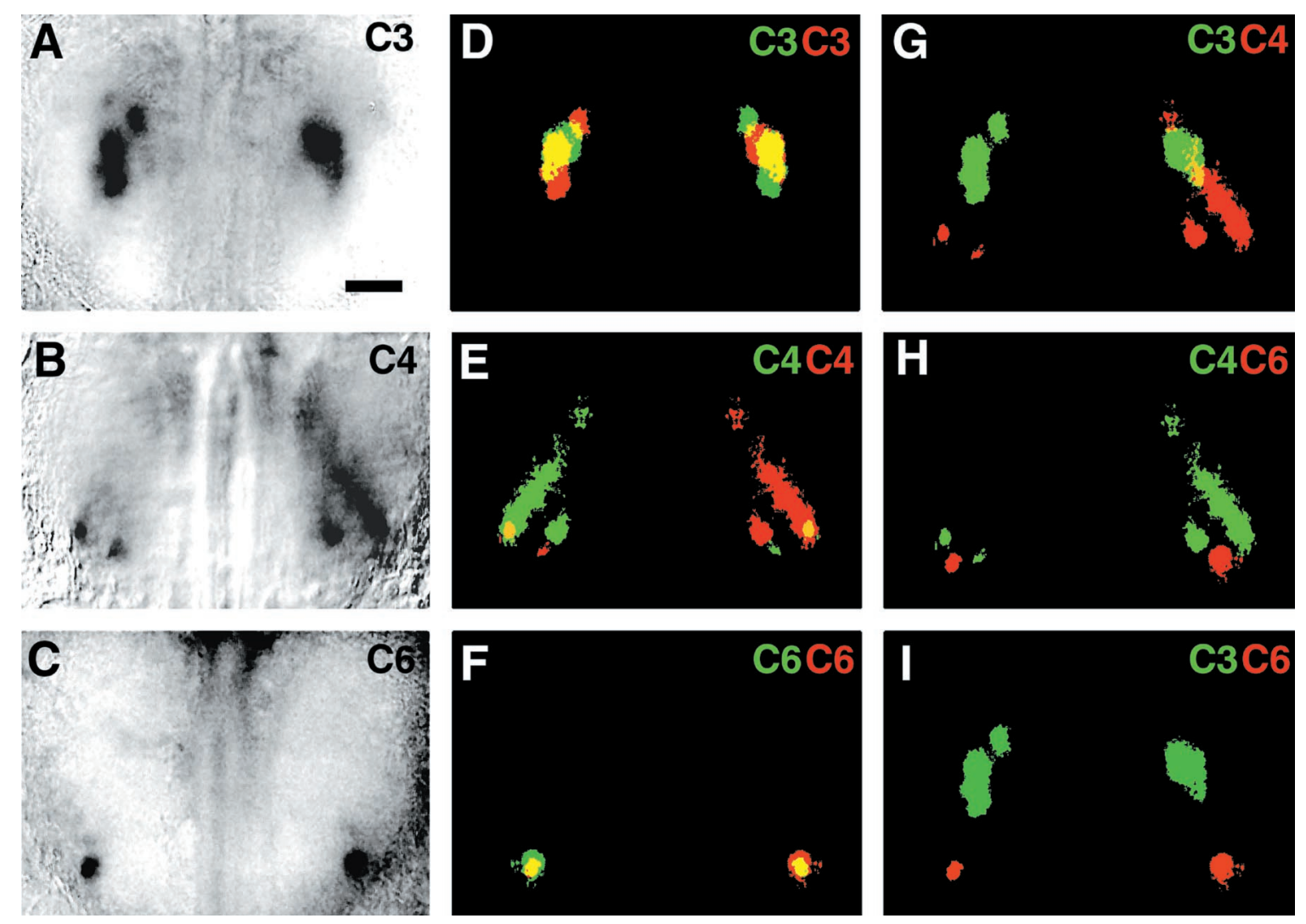

Figure 2. Bilateral symmetry of functional representations. $A-C$, Dorsal views of both olfactory bulbs in an adult mouse, imaged through thinned bone. Bilateral olfactory maps were evoked by $1 \%$ propanal $(A), 1 \%$ butanal $(B)$, and $1 \%$ hexanal $(C)$. Responses to all three odorants activate roughly corresponding regions in the two hemispheres; in the case of hexanal $(C)$, an apparently corresponding glomerulus was activated in each hemisphere. In all panels, caudal is at the top of the panel. $D-F$, Duplicate maps of images $(A-C)$, respectively, in which one copy has been flipped horizontally, pseudocolored (original is red and inverted copy is green), and superimposed on the original. Overlapping regions of activity are yellow. Activity patterns show only partial overlap, indicating that in general they are not precise mirror images. $G$, Color-coded activity maps comparing active regions for propanal (green) superimposed on butanal map (red) reveal that odorants which differ by only one carbon show some overlapping regions (yellow). $H$, $I$, Superimposed activity maps for odors differing by two carbons ( $H$, butanal, green; hexanal, red) or three carbons ( $I$, propanal, green; hexanal, red) show no overlap. Scale bar (shown in $A$ for $A-I$ ): $500 \mu \mathrm{m}$.

thresholded at 2 SD to highlight any differences between the experimental mixture image and the calculated mixture image. Images were then median filtered and imported into Adobe Photoshop for display.

\section{RESULTS}

\section{Optical imaging in the mouse olfactory bulb}

To visualize the activity patterns induced by single odors in mouse olfactory bulbs, we used optical imaging of intrinsic signals as described previously for the rat (Rubin and Katz, 1999) (see Materials and Methods). In anesthetized mice the bone over the entire dorsal surface of both olfactory bulbs $(\sim 3 \times 4 \mathrm{~mm})$ was thinned to transparency. This allowed visualization of $\sim 15 \%$ of the estimated 1800 glomeruli present in each bulb (Royet et al., 1988) but a significantly higher proportion of those receiving input from Zone I, the most dorsal zone of receptor expression in the olfactory epithelium (Ressler et al., 1993; Mori et al., 1999). The size of the mouse enabled us to obtain high-resolution images of both olfactory bulbs simultaneously. Odorants consisted of the well studied series of aliphatic aldehydes of varying carbon chain number (3-10 carbons) (Hatanaka et al., 1992; Imamura et al., 1992; Sato et al., 1994).

\section{Bilateral symmetry of functional maps}

After odorant presentation, activated regions in the bulb appeared primarily as discrete, dark, roughly circular zones sim- ilar in size and shape to those observed in intrinsic imaging studies of the rat olfactory bulb (Fig. 1). Diff use zones of lower intensity, adjacent to the circular regions, were also activated. The circular regions corresponded to individual glomeruli, as verified by subsequently staining the bulb with RH414, a voltage-sensitive styryl dye that vitally stains mouse olfactory glomeruli (Fig. 1) ( $n=3$ mice) (LaMantia et al., 1992; Bozza and Kauer, 1998).

To determine the degree of bilateral symmetry in the two olfactory bulbs of the same individual, bilateral activity maps were collected after stimulation by propanal, butanal, and hexanal ( $n=52$ maps from 37 mice, all at $1 \%$ stimulus concentration). Each map consisted of clusters of as few as two (hexanal) to more than six (butanal) distinct glomeruli, along with less discrete regions of lower activation. Even casual inspection revealed striking bilateral symmetry (Fig. $2 A-C$ ).

Although the activated regions in the maps were clearly symmetric, they were not exact mirror images of one another. The active regions in individual activity maps (defined as those with signals $>2$ SDs above the mean pixel value) were pseudocolored, and duplicate mirror image maps were superimposed on the originals (Fig. 2D-F). To maximize the extent of overlap, we allowed the mirror-image maps to be rotated $\pm 5^{\circ}$ and displaced $\pm 100 \mu \mathrm{m}$ medially and laterally. The ratio of the number of 
A

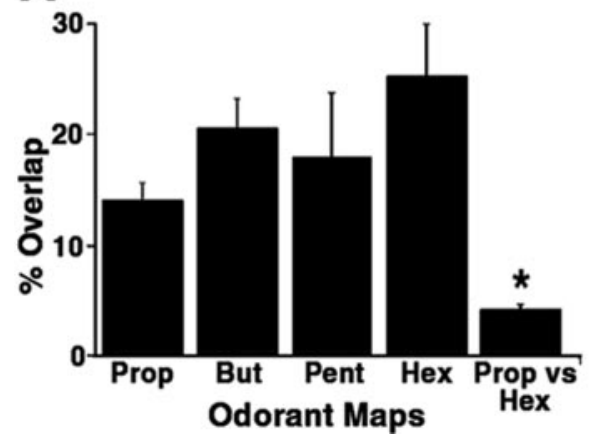

B

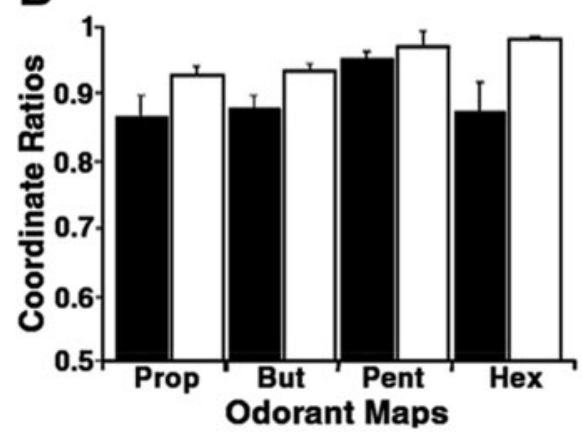

C

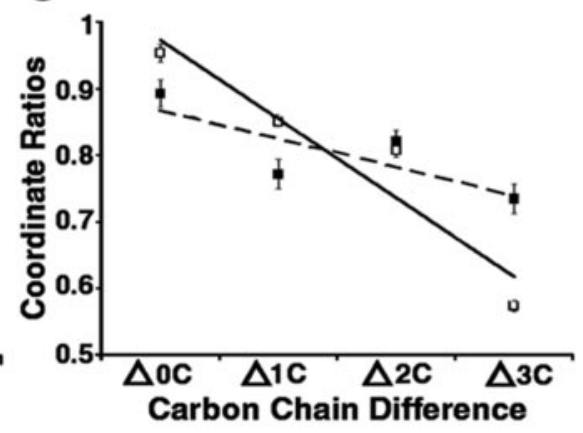

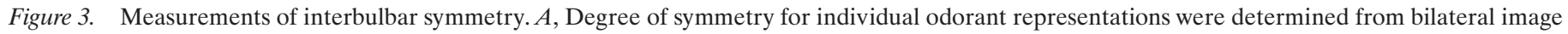

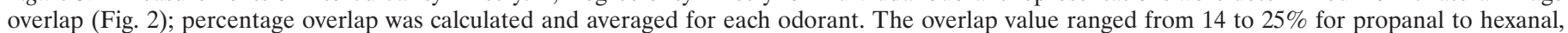

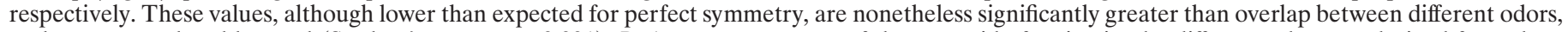

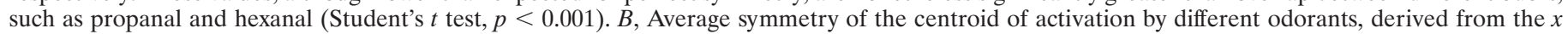

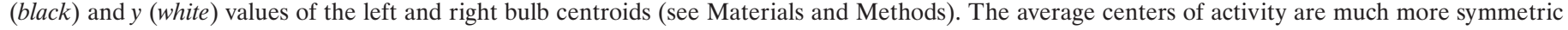

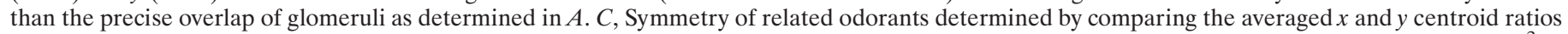

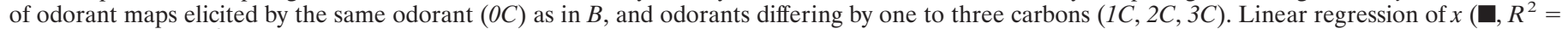

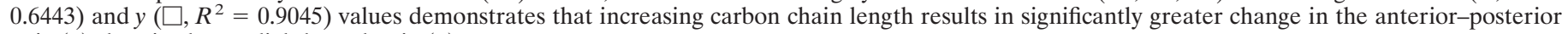
axis $(y)$ than in the medial-lateral axis $(x)$.

overlapping activated pixels to the total number of activated pixels in each map was calculated. If the maps were perfectly bilaterally symmetric, $100 \%$ of the pixels would overlap. Actual maps for all odorants tested overlapped 14-25\% (mean $=19 \pm$ $2 \% ; n=52$ pairs of maps in 37 animals) (Fig. $3 A$ ), which was considerably lower than predicted for exact symmetry. For comparison, we assessed the extent of overlap between maps of related odorants. For example, when the map of propanal was superimposed on the map of hexanal from the same animal, the overlap was $<5 \%$ (Fig. $3 A$ ).

Because the precise location of the active peaks between the two bulbs in response to an odorant varied, we considered whether the "average active center" or centroid of all the peaks within a bulb that respond to a specific odorant showed greater consistency between the two bulbs. To determine whether corresponding regions of each bulb were activated, as opposed to the precise patterns of glomeruli, we determined the centroid of all active regions for each member of the pair of maps in a single animal (see Materials and Methods). We then determined the displacement of each pair of centroids from the midline $(x)$ and from the posterior extent of the imaged region $(y)$ and calculated their ratio (Fig. 3B). If the centroids were located at exactly corresponding, mirror symmetric points, the $x$ and $y$ ratios would be 1 . For all pairs of maps analyzed ( $n=53$ in 31 animals), the average $x$ ratio was $0.89 \pm 0.02$, and the average $y$ ratio was $0.95 \pm$ 0.01 . In contrast, the symmetry ratio for pairs of maps of different odorants in the same animals (i.e., butanal vs hexanal, pentanal vs hexanal) yielded ratios that decreased sharply with increasing difference in carbon chain length (Fig. 3C). The difference was most prominent along the $y$-axis. Because the centroids were all located within a narrow rostrocaudal strip, (see below) differences in the $x$-axis were less pronounced. Thus, regions of the bulb activated by a given odorant showed strong bilateral symmetry, although the exact number and position of activated glomeruli varied.

\section{Stereotypy and variability of odorant representations between animals}

Epithelial neurons bearing the same odorant receptor project to glomeruli at conserved topographic loci in different animals
(Ressler et al., 1994; Vassar et al., 1994; Mombaerts et al., 1996; Wang et al., 1998), although the precise relationships between termination patterns of axons bearing the same receptor can show considerable variability within a local topographic region (Strotmann et al., 2000). To assess whether maps of odorant-evoked
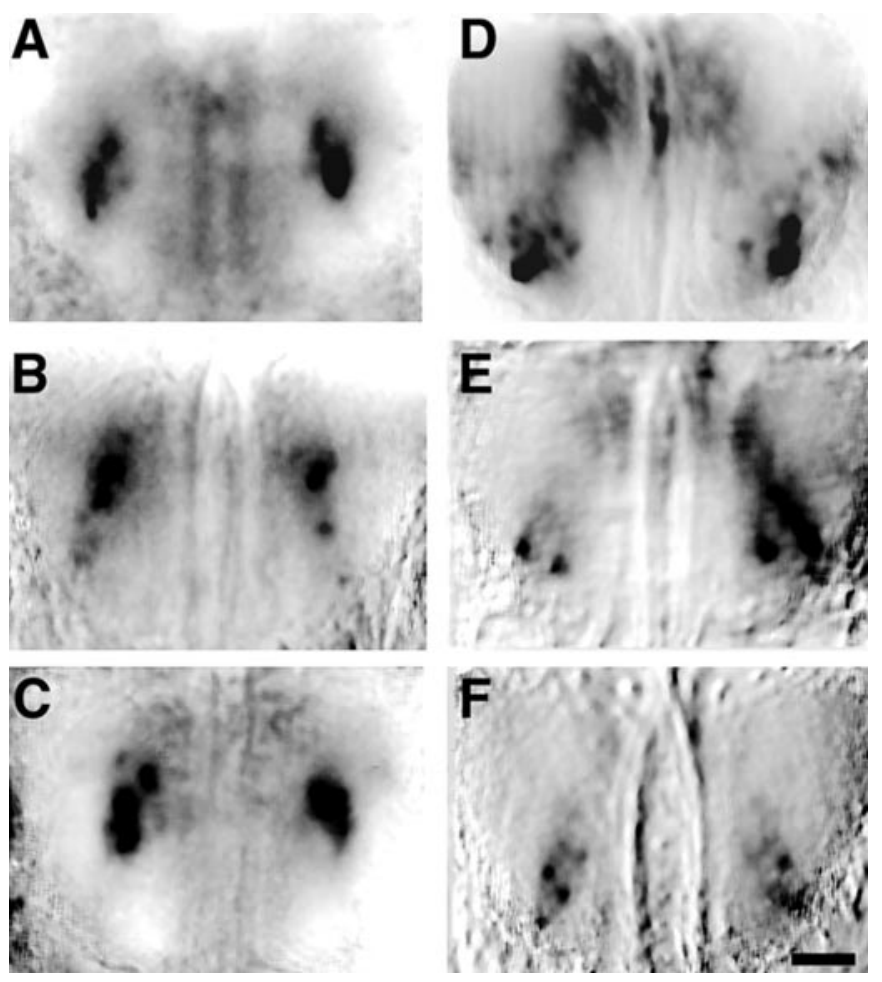

Figure 4. Stereotypy of odorant maps. $A-C$, Bilateral dorsal view of optical maps of both bulbs from three different mice in response to $1 \%$ propanal reveal similar patterns. All maps contain a region of dense activation of several glomeruli near the center of each bulb. $D-F$, Odorant maps evoked by $1 \%$ butanal in three different mice demonstrate a similar resemblance to one another. Each map consisted of two to three highly active glomeruli in a more rostral-lateral region of the dorsal bulb. Caudal is toward the top of each panel. Scale bar, $500 \mu \mathrm{m}$. 

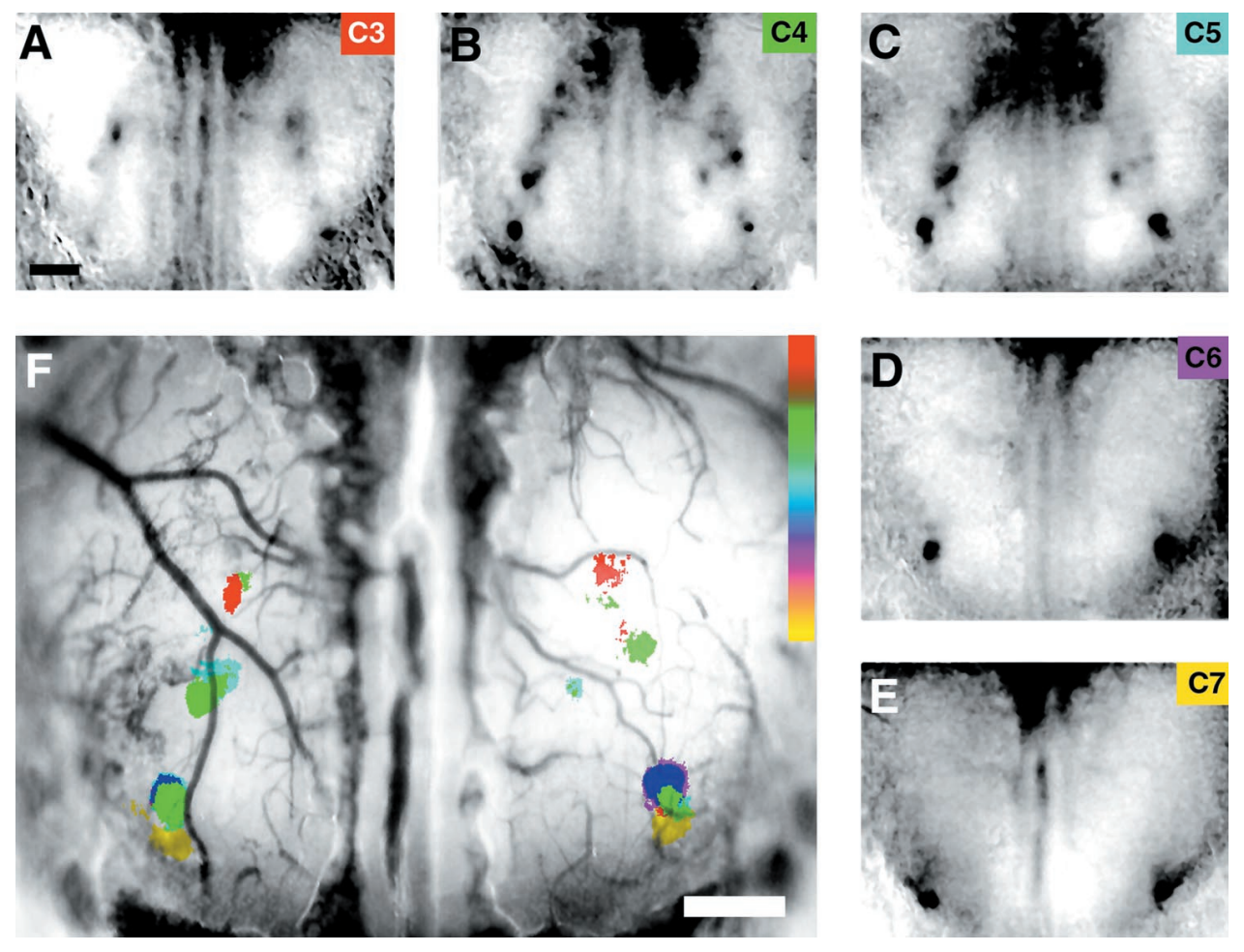

Figure 5. Topographic representation of odorants. $A-E$, Bilateral odorant maps of the response to a series of aliphatic aldehydes (1\%, 3-7 carbons, as noted at the top right-hand corner of each panel). All maps were derived from the same mouse. Regions of highest activity move progressively rostral and lateral with increasing carbon chain length. The resemblance of each map to its nearest neighbor is apparent. $F$, Composite image in which each map has been color coded and superimposed on a single olfactory bulb image containing the blood vessel pattern for the imaged mouse. Structurally related odorants evoke overlapping patterns of activity along a caudal-medial to rostral-lateral strip of both bulbs. Intermediate colors in the spectrum represent overlap of adjacent color maps. Caudal is toward the top of each panel. Scale bars, $500 \mu \mathrm{m}$. activity show a corresponding similarity, we compared the responses to propanal and butanal in 16 mice. At the stimulus concentration used, the number of discrete active glomeruli (determined by thresholding each image at $2 \mathrm{SD}$ above the mean pixel value) fell into a narrow range: $6 \pm 0.4$ (mean \pm SEM) for butanal (range 3-9) and $4 \pm 0.4$ for propanal (range $2-7$ ). The activated glomeruli in different individuals occupied similar locales on the bulbar surface (Fig. 4). This stereotypy was also evident when the topography of these representations was examined in greater detail (see below).

\section{Topography of structurally related odorants}

In the visual, somatosensory, and auditory systems, peripheral sense receptors project topographically to more central structures, thereby preserving neighborhood relationships present in the periphery. Molecular mapping studies show that this is clearly not the case in the olfactory system, but several observations suggest the presence of "odotopic" maps (Yoshihara and Mori, 1997). Olfactory receptor neurons expressing genetically similar odorant receptors project to similar loci in the bulb, and in rats, functional maps for structurally related compounds are adjacent (Rubin and Katz, 1999; Tsuboi et al., 1999; Uchida et al., 2000). From the results described above, it is clear that in the mouse bulb the representations of individual odorants occupy distinct, predictable loci on the dorsal surface of the bulb and that different but related odorants occupy adjacent overlapping regions. Using the larger exposed area in the mouse, and the ability to image bilaterally, we examined the topographic relationship among activity maps for the series of aliphatic aldehydes with three to seven carbons.

Complete odor series maps were obtained in four animals; each map was the result of at least 10 stimulus presentations. Activated regions in response to each odorant were assigned a different color code, and the maps were superimposed. These maps exhibited clear topography. As carbon chain length increased, the activated regions moved anteriorly and laterally (Fig. 5), defining an elongated, narrow strip of activity extending from the midventral to the anterior-lateral edge of the exposed portion of each olfactory bulb. For each map, we determined the active peaks as described above, then located the centroids as a method for defining a single point center of activity within each bulb. We then measured the distance between the active centers elicited by each successive odorant in the series. For each carbon added, the center of activity moved an average $279 \pm 87 \mu \mathrm{m}$, although there was evidence for abrupt jumps rather than smooth changes, as discussed below. Thus, at least for aliphatic aldehydes, a well defined odotopic map is present in the olfactory bulb.

\section{Population topography}

Odor maps from 10 representative individuals were aligned using the posterior border of the bulbs, the midline, and the posterior olfactory bulb sinus as landmarks. The centers of each active region were marked and compiled onto consensus maps for each odor (Fig. 6A). The consensus representation of single odorants occupied $<10 \%$ of the exposed region. Because the exposed region is $\sim 10-15 \%$ of the entire active surface of the main olfactory bulb, only a small region is activated by a single odorant. Given the variability of maps in the two bulbs within individuals (see above), the known variability in glomerular patterns, and the difficulty of precisely aligning maps between animals, the conserved location of these representations is impressive.

Despite the many sources of variability, these data reveal a clear cluster of active regions for each odorant. In the case of propanal and butanal (C4), whose consensus regions partially overlap (C3) (Fig. 6B) the individual odor maps contain discrete glomeruli associated exclusively with one odorant, whereas a subset is activated by both. Consensus regions for butanal (C4) and pentanal (C5) overlap extensively; a greater proportion of their odor maps contain glomeruli activated by both odorants. The same relationship is true for hexanal (C6) and heptanal (C7). 

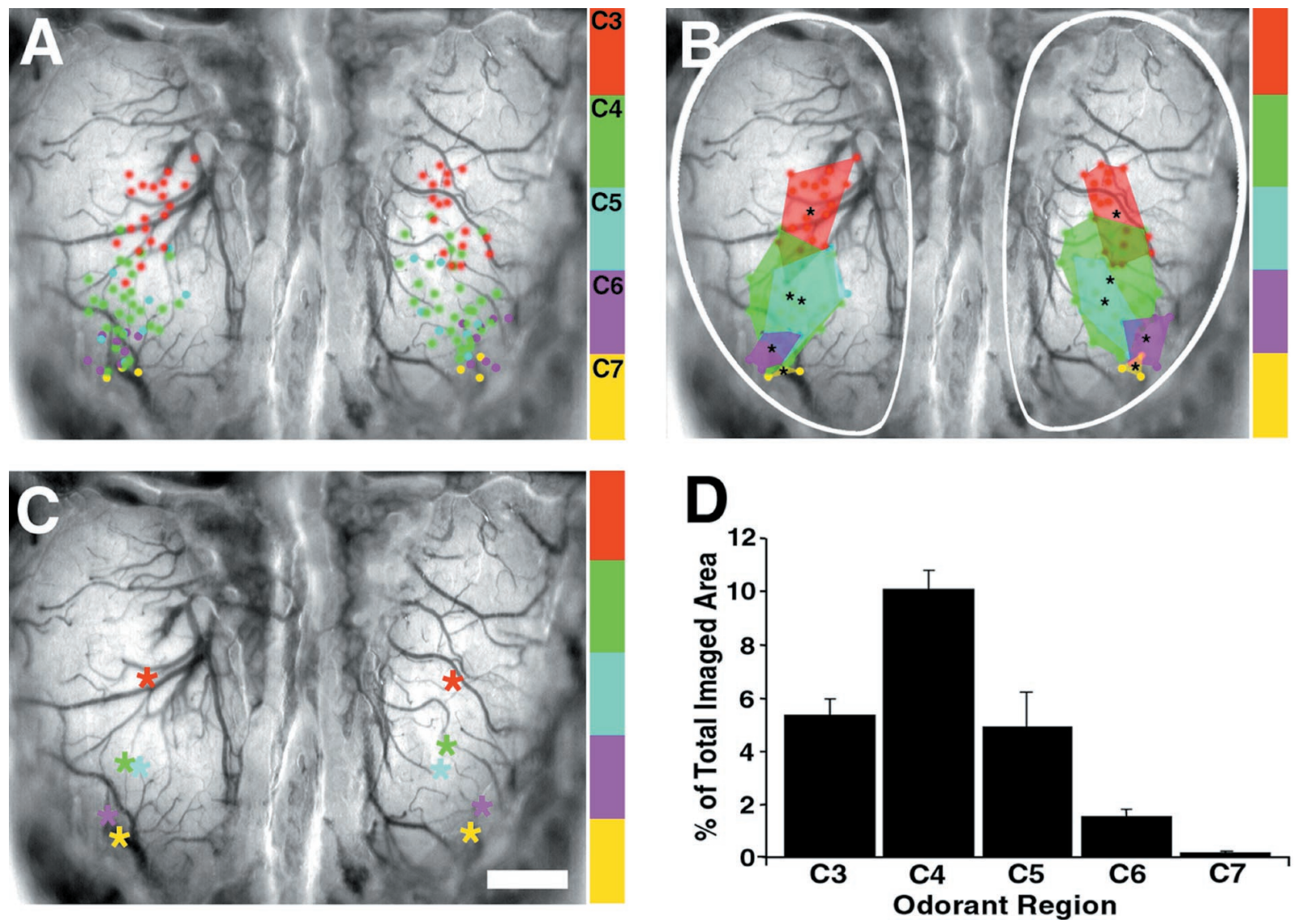

Figure 6. Population topography demonstrates clustering, symmetry, and overlap of odor responsive regions. A, Composite map depicting the active centers from 43 different odorant maps in response to the aliphatic aldehyde odorants containing three to seven carbons (1\% concentration), color coded for odorant, and superimposed on a single olfactory bulb, blood vessel image. In the aggregate image, active centers for each odorant are clustered in distinct but partially overlapping regions. $B$, Perimeters encompassing each odorant cluster depicted in $A$ illustrate the consensus regions of activity for individual odorants and the overlap with consensus regions of neighboring odorants. Note the rostral-lateral progression of active regions as odorants increase in carbon chain length. $C$, Centroids of the activated regions for each odorant-responsive region depicted in $B$ plotted onto a composite map demonstrate symmetry of odorant maps at a population level. $D$, Area of each odorant-responsive region from both bulbs expressed as a percentage of the entire imaged bulb area (outlined by the white trace in $B$ ). Scale bar, $500 \mu \mathrm{m}$.

We believe that some odorants may share more glomeruli in local regions, giving rise to saltatory movement of representations rather than a smooth progression. The commonalties of certain odorants may produce the "pairing" of centroids observed in these representations (Fig. 6C).

\section{Maps of odorant mixtures}

Most imaging and electrophysiological investigations have focused on the central representations of individual odorant molecules. However, odors in the natural environment are typically composed of multiple molecular components. As a first step in understanding how odorant mixtures are represented in the olfactory bulb, we examined olfactory maps collected sequentially from the same animal in response to $1 \%$ propanal $(C 3)$ (Fig. $7 A$ ), followed by $1 \%$ butanal $(C 4)$ and then a two-component mixture of $1 \%$ propanal and $1 \%$ butanal. We specifically sought to determine whether at this first stage of olfactory processing a mixture would elicit a pattern of glomerular activation that was distinct from the sum of those elicited by its two components.

The two-component mixture generated robust activation of the bulb, and visual inspection revealed that the activity map for the binary odor mixture was a simple combination of the two individual odor maps (Fig. 7); glomerular activity was not lost nor were new glomeruli recruited. To more accurately compare mix- ture maps, we next randomly interleaved all three stimuli within the same experiment. So as not to conflate genuine changes in the activity pattern resulting from interactions between the two odors with effects caused simply by increasing stimulus concentration, we examined activity maps in the bulb in response to $2 \%$ butanal, $2 \%$ propanal, or the mixture of $1 \%$ of each.

Although spatial patterns of activity appeared unchanged when odors were presented in combination, the intensity of individual activity peaks was sometimes altered when compared with the corresponding peaks for individual odor maps. Electrophysiological data indicate that odorants which share more molecular features affect the response profiles of mitral cells more strongly than those that are less similar (Mori et al., 1984; Imamura et al., 1992; Katoh et al., 1993; Mori, 1995; Yokoi et al., 1995). To assess the significance of these observations, experimentally acquired mixture maps were compared with a calculated mixture map derived by adding the above-background pixel values for each individual map (Fig. 8) $(n=21)$. We then subtracted the mathematical sum map from the experimentally derived map (Fig. $8 E, F)$. Presenting odorants as a mixture produced no significant change in signal intensity of individual regions of activity. Therefore, activity maps of binary odorant mixtures can be derived directly from the addition of their component maps without 

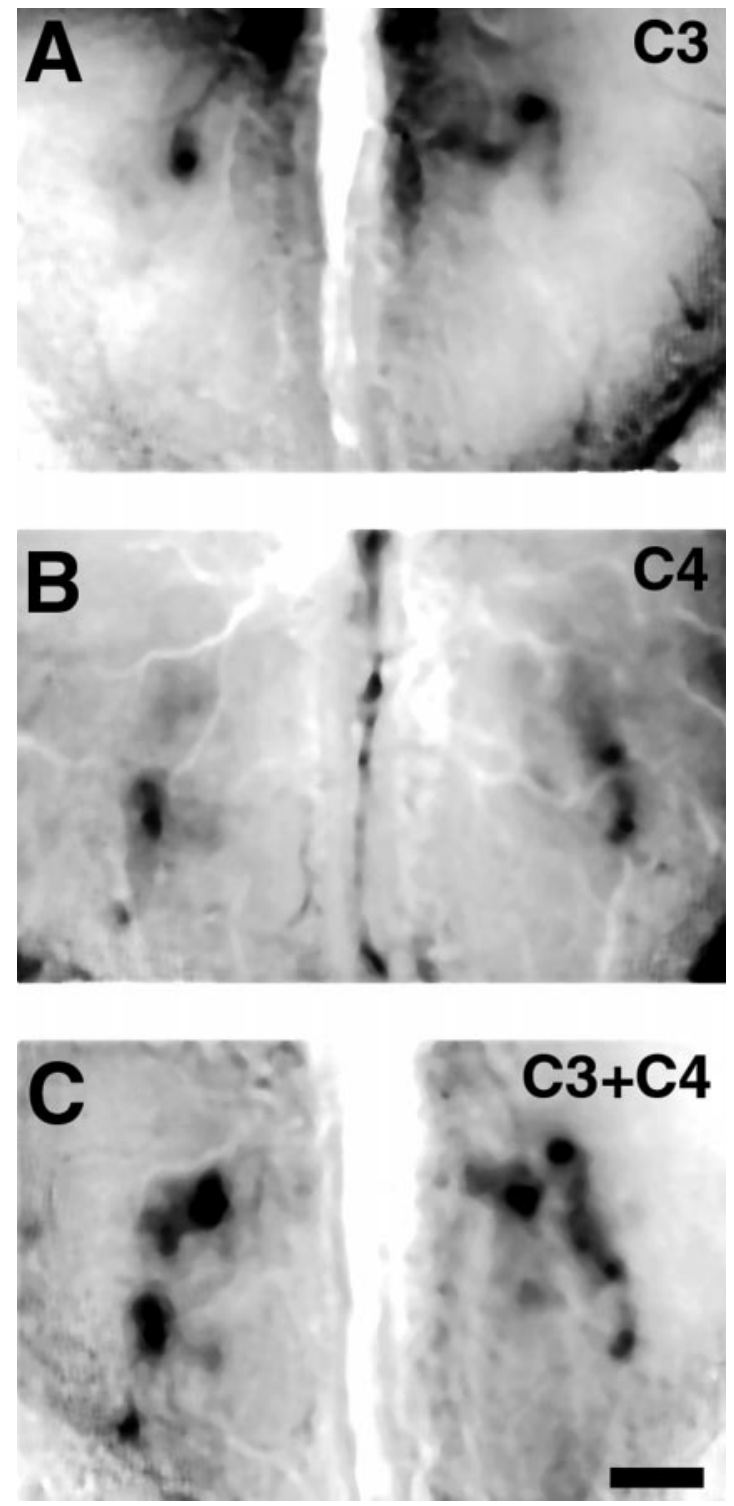

Figure 7. Representation of simple binary mixtures. A, Propanal (1\%) activates two discrete glomeruli on the right bulb and one on the left. An additional activated region is visible in the rostral region of the right bulb. $B$, Optical map of odorant response to $1 \%$ butanal reveals two active peaks in each bulb located in a more rostral-lateral region, with some residual activity detected more caudally. $C$, Optical map of the olfactory bulb response to an equal mixture of $1 \%$ propanal and $1 \%$ butanal reveals an additive pattern of activity. Each of the features detected within the individual odorant maps $(A$ and $B)$ are represented within the mixture map. Regions of lower activity also appear to be additive, suggesting that they represent specific lower level activity and not nonspecific background. Maps were collected sequentially from the same mouse. Caudal is toward the top of the images. Scale bar, $500 \mu \mathrm{m}$.

obvious addition or deletion of individual active regions or without significantly altering the degree of activity within the activated glomeruli.

\section{DISCUSSION}

The sophisticated genetic manipulations possible in mice make them the logical platform for understanding olfactory development, function, and plasticity. Here we have shown that the patterns of functional activation of olfactory receptors observed in mice reflect the most salient features of the molecular maps of receptor organization, including the similarities between individuals, the bilateral symmetry of projections, and the clustering of projections of related receptors (Ressler et al., 1994; Mombaerts et al., 1996; Strotmann et al., 2000). In addition, our data are consistent with similar work in other species using optical imaging (Uchida et al., 2000) or 2-deoxyglucose labeling (Johnson et al., 1999; Johnson and Leon, 2000a,b), demonstrating that odorants of similar molecular structure activate topographically adjacent regions, providing for a topographic organization based on odorant structure.

\section{Bilateral symmetry of odorant maps}

In zebra-fish, honeybees, and fruit flies, which have only a small fraction $(2-5 \%)$ of the number of glomeruli that mice have, the arrangements of both glomeruli and odorant-evoked activity patterns are highly bilaterally symmetric (Baier and Korsching, 1994; Friedrich and Korsching, 1997; Joerges et al., 1997; Gao et al., 2000; Vosshall et al., 2000). Previous studies in rats using 2-DG labeling suggested a similar organization, as did a previous optical imaging study (Rubin and Katz, 1999). However, the ability to simultaneously image both bulbs in mice revealed that odorantactivated glomeruli comprise zones that are highly symmetric on the two sides yet (unlike zebrafish, honeybees, or fruit flies) are not mirror images of one another.

In previous molecular studies, the glomeruli corresponding to individual olfactory receptors were visualized and appeared strikingly symmetric between the two bulbs (Ressler et al., 1994; Vassar et al., 1994; Mombaerts et al., 1996). In a recent report (Strotmann et al., 2000), the termination patterns of several different but closely related olfactory receptors were analyzed in greater detail, revealing that even within the two bulbs of the same animal, nearest-neighbor relationships vary considerably. The compounded effects of this local molecular variability would explain the high degree of symmetry between the centroids of activated regions in the two bulbs but the absence of true mirror symmetry. In some cases (Fig. $2 A-C$ ), the size, shape, position, and degree of activation of a particular glomerulus seemed to have an obvious counterpart in the other hemisphere, but this could not be ascertained directly.

\section{Stereotypy}

Molecular studies have also indicated a remarkable similarity in the number and positions of glomeruli between different animals. Such stereotypy is clearly evident in zebrafish, fruit flies, and honeybees. Considerable functional stereotypy has also been shown in rats using metabolic labeling (Johnson et al., 1998). We observed an imprecise stereotypic map. Although individual mice showed considerable variability in the number and intensity of activated glomeruli, these invariably occupied a limited region of the dorsal surface of the bulb. Functional representations are subject to potential modifications of underlying synapses by differential experience (Purves and Lichtman, 1980) and may therefore demonstrate greater variability among animals than do molecular maps. This is supported by the finding that experience clearly modifies the overall size of the bulb (Brunjes, 1994). Greater insight may come from studies focusing on the variability of representations in different mouse strains, particularly those with known deficits in perceiving certain odorants. For example C57BL/6 and NJB/BINJ mice have greatly reduced sensitivity to isovaleric acid and androstenone, respectively, which may be reflected in substantially different maps between the two strains (Wang et al., 1993; Griff and Reed, 1995). 

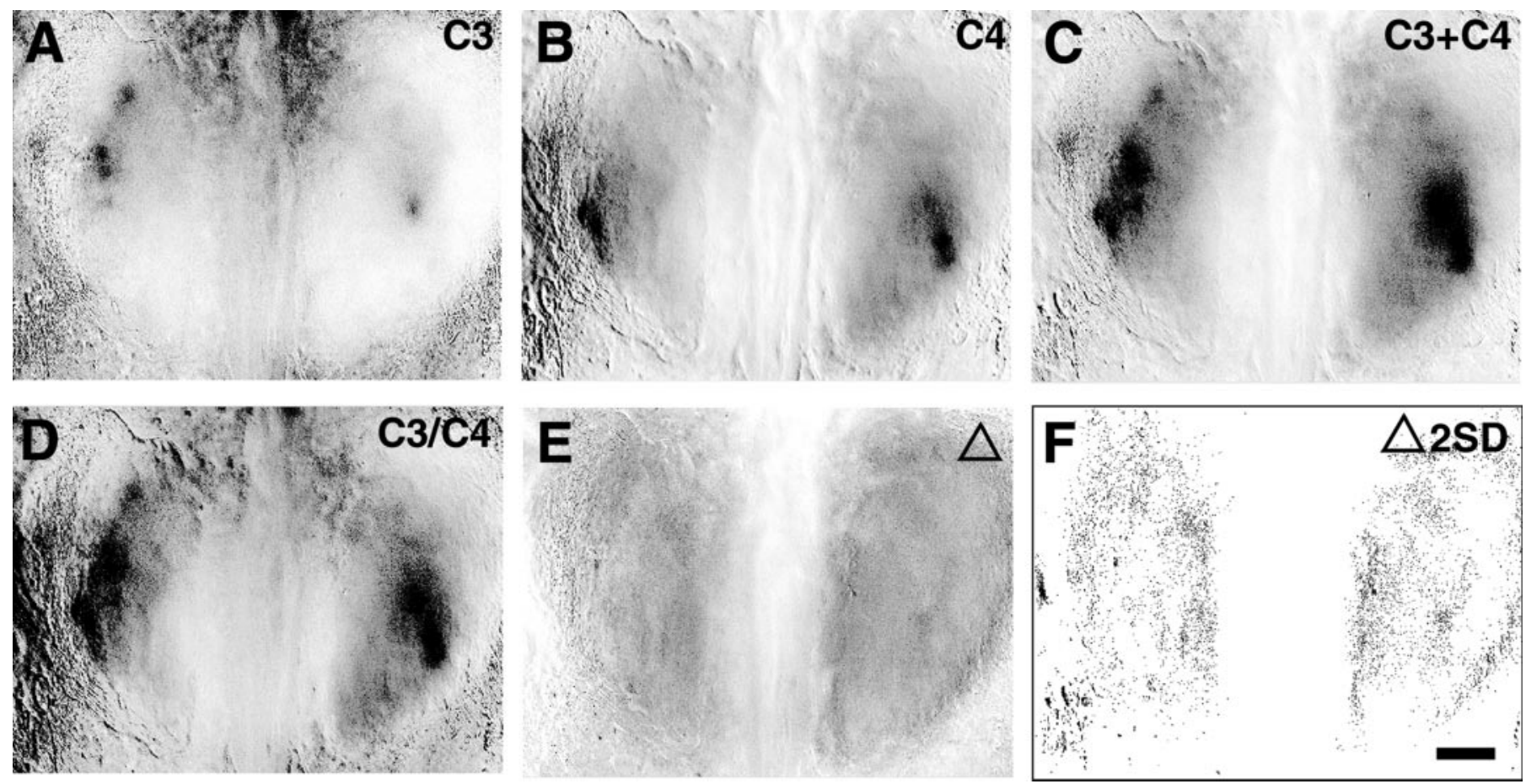

Figure 8. Binary mixtures are the quantitative sum of individual odorant maps. $A-C$, Set of optical maps collected within a single interleaved experiment showing response patterns to $2 \%$ propanal $(A), 2 \%$ butanal $(B)$, and a mixture of $1 \%$ of each $(C)$. $D$, Calculated odorant mixture map derived by summing the two individual odorant maps in $A$ and $B$. E. Calculated difference map derived by subtracting the calculated mixture map $(D)$ from the experimentally derived mixture map $(C) . F$, The difference map, in which all pixels $>2$ SD difference from the mean are black, reveals no significant quantitative difference between the experimental and mathematically derived maps, demonstrating that the activity map produced by this binary mixture is predicted by the sum of the two independent components. Scale bar, $500 \mu \mathrm{m}$.

\section{Topography of odorant maps}

In the visual, somatosensory, and auditory systems, cortical and subcortical structures contain topographic maps of the peripheral receptor surface in which neighborhood relationships are largely preserved. This is clearly not the case in the olfactory system, because receptor neurons expressing different odorant receptors are completely intermingled with the epithelium. However, in the olfactory bulb, axons bearing receptors with a high degree of molecular similarity converge on nearby glomeruli (Tsuboi et al., 1999). Furthermore, as we have observed here, individual odorants tend to activate clusters of nearby glomeruli, rather than widely scattered individual glomeruli. Coupled with these observations, the progressive shift in aldehyde representations suggests that the topography of odorant receptors is mirrored by a functional topography as well. A recent extensive study in rats (Uchida et al., 2000) suggests that this organization extends to different classes of odorant molecules as well, implying that the regular shifts in activated glomeruli are not simply a quirk of the aldehyde representations.

Similar studies in rats show aldehyde activation of a region in the anterior-medial portion of the bulb and a progressive shift in representations anteriorly and laterally with increasing carbon chain number (Johnson et al., 1998; Rubin and Katz, 1999; Uchida et al., 2000). The topography of aldehyde representations in mice appears comparable (Fig. 5) with a general shift from the posterior-medial area of the bulb to the anterior-lateral. However, compared with rats, the activated glomeruli in mice are located somewhat more laterally relative to the midline. We also observe that the orientation of this aldehyde-responsive strip is perpendicular to the molecular axis of symmetry described by several laboratories (Ressler et al., 1994; Vassar et al., 1994), suggesting that a similarly organized aldehyde-responsive strip may exist on the ventral surface of each bulb, as predicted by 2-DG studies (Johnson et al., 1999; Johnson and Leon, 2000a,b).

\section{Functional zones in the olfactory bulb}

Receptors that share a high degree of molecular similarity project to neighboring glomeruli in the olfactory bulb (Wang et al., 1998; Tsuboi et al., 1999), but the specific location of a particular glomerulus is not precisely fixed relative to its neighbors (Strotmann et al., 2000). The extension of these principles to a small population of glomeruli suggests that the specific location of a glomerulus is not stereotyped but can shift within the confines of a defined zone. This model is consistent with functional data demonstrating that a particular odor activates glomeruli within a specific region of the bulb, but considerable variability exists within that region. Moreover, related odorants activate neighboring, even overlapping regions of the olfactory bulb. By compiling many olfactory maps we outlined the consensus boundaries of these regions, defining functional zones for the aldehyde series of odorants. Given the similarity between molecular and functional topography, the regions that we observe may also correspond to developmentally restricted zones for establishing molecular topography. It will be interesting to compare these zones with the molecular topography of the dorsal olfactory bulb once the local variability has been similarly mapped.

\section{Representation of binary mixtures}

Most natural odorants consist of complex mixtures of many distinct components, each of which would be expected to activate 
distinct patterns of glomeruli, yet they are often perceived as single odorants. How then are they represented within the olfactory bulb? Electrophysiological and psychophysical studies in several species reveal various types of nonlinear interactions that occur in response to odorant mixtures (Derby et al., 1991a,b; Getz and Akers, 1995; Olson and Derby, 1995). These interactions may underlie behavioral observations, such as odor masking and overshadowing (Laing et al., 1989; Derby et al., 1996; Linster and Smith, 1997; Pelz et al., 1997). Lateral inhibition in the bulb can sharpen the responses of neurons to one odorant by inhibiting responses to molecularly similar odorants (Yokoi et al., 1995). There may also be synergistic effects in which the response of an olfactory bulb neuron to a mixture may be more than the sum of its response to the individual components. Thus, we sought to determine whether any such interactions could be observed in response to stimulation by a binary mixture.

At the level of analysis possible with maps derived from optical imaging, glomerular patterns in response to a simple odorant mixture could be accounted for by addition of the maps of each individual component. When balanced for the total amount of odorant presented, we did not observe evidence for novel glomeruli that were specifically activated only by the mixture, nor did we observe glomeruli activated by either component that were absent when the mixture was presented.

These findings are consistent with human psychophysical studies demonstrating that on average the individual components in mixtures of up to four odorants can be discriminated (Livermore and Laing, 1996; Cain et al., 1998; Livermore and Laing, 1998). However, human studies also reveal that individual components are perceived as less intense when presented in mixtures, which correlates with behavioral paradigms such as overshadowing (Rescorla, 1980; Linster and Smith, 1997). These data suggest that mixtures modify sensory processing, which may or may not occur at the level of the olfactory bulb. Thus, although the patterns of activated glomeruli appear to be additive, the actual output from mitral cells to more central structures might be very different (Vickers et al., 1998; Christensen et al., 2000). Using optical patterns of activation as guides, however, it will be possible to directly target extracellular and intracellular recordings to specific groups of mitral cells to determine more precisely whether and how response profiles to mixtures differ from that of individual components.

\section{REFERENCES}

Baier H, Korsching S (1994) Olfactory glomeruli in the zebrafish form an invariant pattern and are identifiable across animals. J Neurosci 14:219-230.

Bozza TC, Kauer JS (1998) Odorant response properties of convergent olfactory receptor neurons. J Neurosci 18:4560-4569.

Brunjes PC (1994) Unilateral naris closure and olfactory system development. Brain Res Brain Res Rev 19:146-160.

Cain WS, de Wijk R, Lulejian C, Schiet F, See LC (1998) Odor identification: perceptual and semantic dimensions. Chem Senses 23: 309-326.

Christensen TA, Pawlowski VM, Lei H, Hildebrand JG (2000) Multiunit recordings reveal context-dependent modulation of synchrony in odor-specific neural ensembles. Nat Neurosci 3:927-931.

Cinelli AR, Neff SR, Kauer JS (1995) Salamander olfactory bulb neuronal activity observed by video rate, voltage-sensitive dye imaging. I. Characterization of the recording system. J Neurophysiol 73: 2017-2032.

Derby CD, Girardot MN, Daniel PC (1991a) Responses of olfactory receptor cells of spiny lobsters to binary mixtures. I. Intensity mixture interactions. J Neurophysiol 66:112-130.

Derby CD, Girardot MN, Daniel PC (1991b) Responses of olfactory receptor cells of spiny lobsters to binary mixtures. II. Pattern mixture interactions. J Neurophysiol 66:131-139.

Derby CD, Hutson M, Livermore BA, Lynn WH (1996) Generalization among related complex odorant mixtures and their components: analysis of olfactory perception in the spiny lobster. Physiol Behav 60:87-95.

Faber T, Joerges J, Menzel R (1999) Associative learning modifies neural representations of odors in the insect brain. Nat Neurosci 2:74-78.

Friedrich RW, Korsching SI (1997) Combinatorial and chemotopic odorant coding in the zebrafish olfactory bulb visualized by optical imaging. Neuron 18:737-752.

Gao Q, Yuan B, Chess A (2000) Convergent projections of Drosophila olfactory neurons to specific glomeruli in the antennal lobe. Nat Neurosci 3:780-785.

Getz WM, Akers RP (1995) Partitioning non-linearities in the response of honey bee olfactory receptor neurons to binary odors. Biosystems 34:27-40.

Griff IC, Reed RR (1995) The genetic basis for specific anosmia to isovaleric acid in the mouse. Cell 83:407-414.

Guthrie KM, Anderson AJ, Leon M, Gall C (1993) Odor-induced increases in c-fos mRNA expression reveal an anatomical "unit" for odor processing in olfactory bulb. Proc Natl Acad Sci USA 90:3329-3333.

Hatanaka A, Kajiwara T, Horino H, Inokuchi K (1992) Odor-structure relationships in n-hexenols and n-hexenals. $Z$ Naturforsch [C] 47:183-189.

Imamura K, Mataga N, Mori K (1992) Coding of odor molecules by mitral/tufted cells in rabbit olfactory bulb. I. Aliphatic compounds. J Neurophysiol 68:1986-2002.

Joerges J, Kuttner A, Galizia CG, Menzel R (1997) Representations of odours and odour mixtures visualized in the honeybee brain. Nature 387:285-288.

Johnson BA, Leon M (2000a) Modular representations of odorants in the glomerular layer of the rat olfactory bulb and the effects of stimulus concentration. J Comp Neurol 422:496-509.

Johnson BA, Leon M (2000b) Odorant molecular length: one aspect of the olfactory code. J Comp Neurol 426:330-338.

Johnson BA, Woo CC, Leon M (1998) Spatial coding of odorant features in the glomerular layer of the rat olfactory bulb. J Comp Neurol 393:457-471.

Johnson BA, Woo CC, Hingco EE, Pham KL, Leon M (1999) Multidimensional chemotopic responses to n-aliphatic acid odorants in the rat olfactory bulb. J Comp Neurol 409:529-548.

Jourdan F, Duveau A, Astic L, Holley A (1980) Spatial distribution of $\left[{ }^{14} \mathrm{C}\right] 2$-deoxyglucose uptake in the olfactory bulbs of rats stimulated with two different odours. Brain Res 188:139-154.

Katoh K, Koshimoto H, Tani A, Mori K (1993) Coding of odor molecules by mitral/tufted cells in rabbit olfactory bulb. II. Aromatic compounds. J Neurophysiol 70:2161-2175.

Laing DG, Panhuber H, Slotnick BM (1989) Odor masking in the rat. Physiol Behav 45:689-694.

LaMantia AS, Pomeroy SL, Purves D (1992) Vital imaging of glomeruli in the mouse olfactory bulb. J Neurosci 12:976-988.

Lancet D, Greer CA, Kauer JS, Shepherd GM (1982) Mapping of odorrelated neuronal activity in the olfactory bulb by high-resolution 2-deoxyglucose autoradiography. Proc Natl Acad Sci USA 79:670-674.

Leon M, Coopersmith R, Ulibarri C, Porter RH, Powers JB (1984) Development of olfactory bulb organization in precocial and altricial rodents. Brain Res 314:45-53.

Linster C, Smith BH (1997) A computational model of the response of honey bee antennal lobe circuitry to odor mixtures: overshadowing, blocking and unblocking can arise from lateral inhibition. Behav Brain Res 87:1-14.

Livermore A, Laing DG (1996) Influence of training and experience on the perception of multicomponent odor mixtures. J Exp Psychol Hum Percept Perform 22:267-277.

Livermore A, Laing DG (1998) The influence of odor type on the discrimination and identification of odorants in multicomponent odor mixtures. Physiol Behav 65:311-320.

Malnic B, Hirono J, Sato T, Buck LB (1999) Combinatorial receptor codes for odors. Cell 96:713-723.

Mombaerts P, Wang F, Dulac C, Chao SK, Nemes A, Mendelsohn M, Edmondson J, Axel R (1996) Visualizing an olfactory sensory map. Cell 87:675-686.

Mori K (1995) Relation of chemical structure to specificity of response in olfactory glomeruli. Curr Opin Neurobiol 5:467-474.

Mori K, Nowycky MC, Shepherd GM (1984) Synaptic excitatory and inhibitory interactions at distal dendritic sites on mitral cells in the isolated turtle olfactory bulb. J Neurosci 4:2291-2296.

Mori K, Nagao H, Yoshihara Y (1999) The olfactory bulb: coding and processing of odor molecule information. Science 286:711-715.

Olson KS, Derby CD (1995) Inhibition of taurine and 5'AMP olfactory receptor sites of the spiny lobster Panulirus argus by odorant compounds and mixtures. J Comp Physiol [A] 176:527-540.

Onoda N (1992) Odor-induced fos-like immunoreactivity in the rat olfactory bulb. Neurosci Lett 137:157-160.

Pelz C, Gerber B, Menzel R (1997) Odorant intensity as a determinant for olfactory conditioning in honeybees: roles in discrimination, overshadowing and memory consolidation. J Exp Biol 200:837-847. 
Purves D, Lichtman JW (1980) Elimination of synapses in the developing nervous system. Science 210:153-157.

Rescorla RA (1980) Simultaneous and successive associations in sensory preconditioning. J Exp Psychol Anim Behav Process 6:207-216.

Ressler KJ, Sullivan SL, Buck LB (1993) A zonal organization of odorant receptor gene expression in the olfactory epithelium. Cell 73:597-609.

Ressler KJ, Sullivan SL, Buck LB (1994) Information coding in the olfactory system: evidence for a stereotyped and highly organized epitope map in the olfactory bulb. Cell 79:1245-1255.

Royet JP, Souchier C, Jourdan F, Ploye H (1988) Morphometric study of the glomerular population in the mouse olfactory bulb: numerical density and size distribution along the rostrocaudal axis. J Comp Neurol 270:559-568.

Rubin BD, Katz LC (1999) Optical imaging of odorant representations in the mammalian olfactory bulb. Neuron 23:499-511.

Sallaz M, Jourdan F (1993) C-fos expression and 2-deoxyglucose uptake in the olfactory bulb of odour-stimulated awake rats. NeuroReport $4: 55-58$.

Sato T, Hirono J, Tonoike M, Takebayashi M (1994) Tuning specificities to aliphatic odorants in mouse olfactory receptor neurons and their local distribution. J Neurophysiol 72:2980-2989.

Sharp FR, Kauer JS, Shepherd GM (1975) Local sites of activity-related glucose metabolism in rat olfactory bulb during olfactory stimulation. Brain Res 98:596-600.

Sharp FR, Kauer JS, Shepherd GM (1977) Laminar analysis of 2-deoxyglucose uptake in olfactory bulb and olfactory cortex of rabbit and rat. J Neurophysiol 40:800-813.

Skeen LC (1977) Odor-induced patterns of deoxyglucose consumption in the olfactory bulb of the tree shrew, Tupaia glis. Brain Res $124: 147-153$.

Stewart WB, Kauer JS, Shepherd GM (1979) Functional organization of rat olfactory bulb analyzed by the 2-deoxyglucose method. J Comp Neurol 185:715-734.

Strotmann J, Conzelmann S, Beck A, Feinstein P, Breer H, Mombaerts P
(2000) Local permutations in the glomerular array of the mouse olfactory bulb. J Neurosci 20:6927-6938.

Tsuboi A, Yoshihara S, Yamazaki N, Kasai H, Asai-Tsuboi H, Komatsu M, Serizawa S, Ishii T, Matsuda Y, Nagawa F, Sakano H (1999) Olfactory neurons expressing closely linked and homologous odorant receptor genes tend to project their axons to neighboring glomeruli on the olfactory bulb. J Neurosci 19:8409-8418.

Uchida N, Takahashi YK, Tanifuji M, Mori K (2000) Odor maps in the mammalian olfactory bulb: domain organization and odorant structural features. Nat Neurosci 3:1035-1043.

Vassar R, Chao SK, Sitcheran R, Nunez JM, Vosshall LB, Axel R (1994) Topographic organization of sensory projections to the olfactory bulb. Cell 79:981-991.

Vickers NJ, Christensen TA, Hildebrand JG (1998) Combinatorial odor discrimination in the brain: attractive and antagonist odor blends are represented in distinct combinations of uniquely identifiable glomeruli. J Comp Neurol 400:35-56.

Vosshall LB, Wong AM, Axel R (2000) An olfactory sensory map in the fly brain. Cell 102:147-159.

Wang F, Nemes A, Mendelsohn M, Axel R (1998) Odorant receptors govern the formation of a precise topographic map. Cell 93:47-60.

Wang HW, Wysocki CJ, Gold GH (1993) Induction of olfactory receptor sensitivity in mice. Science 260:998-1000.

Xu F, Kida I, Hyder F, Shulman RG (2000) Assessment and discrimination of odor stimuli in rat olfactory bulb by dynamic functional MRI. Proc Natl Acad Sci USA 97:10601-10606.

Yang X, Renken R, Hyder F, Siddeek M, Greer CA, Shepherd GM, Shulman RG (1998) Dynamic mapping at the laminar level of odorelicited responses in rat olfactory bulb by functional MRI. Proc Natl Acad Sci USA 95:7715-7720.

Yokoi M, Mori K, Nakanishi S (1995) Refinement of odor molecule tuning by dendrodendritic synaptic inhibition in the olfactory bulb. Proc Natl Acad Sci USA 92:3371-3375.

Yoshihara Y, Mori K (1997) Basic principles and molecular mechanisms of olfactory axon pathfinding. Cell Tissue Res 290:457-463. 